\title{
Water toxicity monitoring using Vibrio fischeri: a method free of interferences from colour and turbidity
}

\author{
Elsa Correia Faria, Bernard J. Treves Brown and Richard D. Snook* \\ Department of Instrumentation and Analytical Science, UMIST, PO Box 88, \\ Manchester,UKM60 1QD. E-mail: Dick.Snook@umist.ac.uk; \\ Fax: ++44-(0)161-200 4911; Tel: ++44-(0)161-2004893
}

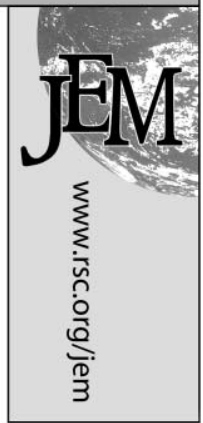

Received 11th September 2003, Accepted 26th November 2003

First published as an Advance Article on the web 20th January 2004

\begin{abstract}
In this paper the kinetic method for the determination of toxicity using Vibrio fischeri is described and suggested as a potential method for the continuous screening of wastewater toxicity. The kinetic method was demonstrated to be free from interferences due to colour and turbidity normally observed when testing wastewater samples with this organism. This is of great importance for the application of the method to remote toxicity screening of wastewaters. The effect of colour, investigated using $50 \mathrm{ppm} \mathrm{Zn}^{2+}$ solutions containing the food-dye tropaeolin $\mathrm{O}$, and the effect of turbidity, investigated using $50 \mathrm{ppm} \mathrm{Zn}^{2+}$ solutions containing white optically reflective and coloured optically absorbing polystyrene beads, is reported. It was also found that the design of the light detection system of the instrument ensures efficient collection of the light scattered by particles in the sample, which enables a greater range of turbid samples to be tested. In addition the natural light decay was found to be negligible during the duration of a 10 min test and thus one channel would be enough to carry out the tests. This would mean halving the quantity of bacterial reagent used and reducing the cost of the tests.
\end{abstract}

\section{Introduction}

It is generally accepted that pollution is a biological phenomenon and that as a consequence only total toxicity can give an indication of an effluent's likely effect on receiving ecosystems. This is now reflected in the changes to the form of the consent licences being granted and enforced by regulatory organisations. Increased emphasis is being placed upon total toxicity as a limiting factor used to control discharges with the objective of improving the quality of receiving watercourses. ${ }^{1}$ Whole Effluent Toxicity (WET) has been successfully implemented in the USA since 1991 and is now a vital component of the water quality standards implementation through the National Pollutant Discharge Elimination System (NPDES) permitting process. ${ }^{2,3}$ In the UK the Environment Agency (EA) aims to introduce a similar scheme under the name of Direct Toxicity Assessment (DTA). DTA is intended as an additional measure of effluent quality in order to provide an early warning system that can be used as a trigger for action to avert pollution incidents by minimising lethal toxicity from point source discharges. ${ }^{2}$ It is believed that DTA will be an easier way to gain environmental benefits from the UK discharge consents system, and that it will ease the load in terms of the number of tests the water monitoring industries will be required to perform because total toxicity characterises the effect of the whole sample while identification of the specific pollutants is not required. ${ }^{4}$

In addition, in the UK wastewater treatment companies treat the discharge from industrial operators along with domestic sewage and road runoff. ${ }^{4}$ As a consequence the composition of effluents can change significantly very rapidly. It is thus necessary to continuously monitor the effluents from the treatment plants to ensure effective protection of the watercourses. It is also important to continuously monitor the influents to the treatment plant to ensure that they are not so toxic as to damage the biological treatment, which would result in major costs to the wastewater treatment companies.

The speed and ease with which results can be obtained with Vibrio fischeri tests, the convenience of the test organisms being available in freeze-dried and liquid-dried form and thus being easily reconstituted and used, coupled to the fact that these tests have been validated and widely used for wastewater and environmental monitoring makes assays that use Vibrio fischeri a good choice for the purpose of continuous remote initial screening. ${ }^{5-8}$

Several commercial tests that use Vibrio fischeri (strain NRRL B-11177) as the test organism are available, these include Microtox ${ }^{\mathbb{R}}$; LUMIStox ${ }^{\mathbb{R}}$; Biotox ${ }^{(\mathbb{m}}$; and ToxAlert ${ }^{\mathrm{R}}$. 8 These tests rely on the measurement of the percent inhibition in the light produced by bacteria caused by the addition of dilutions of the sample to the bacteria after an incubation period (usually 5,15 , or $30 \mathrm{~min}$ ). However, when testing wastewater samples for toxicity using Vibrio fischeri the presence of colour and/or turbidity can cause interferences leading to erroneous results, usually deeming samples more toxic than they are.

The problems experienced when testing wastewater samples using tests based on bioluminescent organisms, arise due to the ability of suspended solids to scatter and/or absorb light causing turbidity, which affects the accurate determination of the amount of light produced by the bacteria. This results in the light intensity measured after incubation being lower than that actually produced by the bacteria. Filtration is not always an option as this can affect the representative nature of the sample. It is also laborious, delays the test, and constitutes an extra source of errors. Like turbidity, true colour (in the following discussion 'colour' refers to 'true colour') present in water, especially due to compounds that absorb around $490 \mathrm{~nm}$ (red, orange, and brown), can cause problems by absorbing light produced by the bacteria thus causing a reduction in the light intensity measured after the incubation period which is not due to the toxicity of the sample. Consequently, coloured and/or turbid samples will be deemed more toxic than they really are. For this reason tests that rely upon discrete measurements of light levels from bacteria at particular times in the analysis cycle can suffer badly from the presence of high levels of suspended solids and colour.

The Microtox ${ }^{\mathbb{B}}$ test does have a colour correction procedure 
which can attenuate the interferences observed for coloured samples and samples containing optically absorbing particles. However, the procedure is laborious, can constitute an additional source of error, and does not correct for interferences caused by white optically reflective particles. ${ }^{9}$ The alternative protocol adopted here is the measurement of toxicity via a kinetic measurement of the decay in light produced by Vibrio fischeri when it is challenged with solutions containing toxic compounds. As well as possessing the advantages of the commonly used methods, this protocol has been predicted to be free from the effects of suspended solids and the presence of colour and consequently to be applicable to test coloured and turbid samples in the same way as clear samples. ${ }^{10}$ This hypothesis is tested in the work reported here. The freedom from interferences due to colour and turbidity makes this a very promising method to be used for continuous screening of wastewaters, particularly because it is possible to be completely automated.

Furthermore, it was also found that the light produced by unchallenged bacteria remained constant for the first $1000 \mathrm{~s}$ of the tests and that it then decays following first order kinetics. This makes blank correction unnecessary and hence a single channel is sufficient to carry out the test. This halves the amount of bacterial reagent used and so reduces the cost of the tests.

\section{The kinetic approach}

The kinetic method uses the marine luminescent bacterium Vibrio fischeri (strain NRRL B-11177) as the test organism, and its capacity to produce light to determine the total toxicity of a sample. The parameter determined by this method is not the common $\mathrm{EC}_{50}$ but a novel one, $\mathrm{ET}_{50} . \mathrm{ET}_{50}$ is the time that it takes the sample to reduce the light produced by the bacteria by $50 \%$, a parameter that could be useful to describe the toxicity of a water sample, especially when only one measurement is required, e.g. for routine screening.

This method is based on the empirical observation that the decay of the light produced by Vibrio fischeri when challenged with a toxin follows first order kinetics. Although the mechanism of toxicity is not generally known this observation seems to be in agreement with the phenomenon first observed by Ikeda in 1897 and which has been often observed since, that the rate of death in populations of individual cells follows first order kinetics. ${ }^{11}$ As no evidence exists that indicates that there are co-operative effects between individual cells that could affect the kinetics this seems to be a reasonable assumption.

When the bacteria are challenged with a toxic sample light production decreases with time, not only due to the toxicity of the sample, but also due to the natural decay. ${ }^{10}$ Therefore, it is necessary to consider two rate constants: the toxic response rate constant $\left(k_{1}\right)$ and the natural decay rate constant $\left(k_{2}\right)$. The rate of decay in the light produced by bacteria challenged with a toxic sample can be described by eqn. (1):

$$
\frac{\mathrm{d} l_{\mathrm{C}, t}}{\mathrm{~d} t}=-l_{\mathrm{C}, t}\left(k_{1}+k_{2}\right)
$$

where $I_{\mathrm{C}, t}$ is the intensity of the light produced by the challenged bacteria at time $t ; t$ is time; $k_{1}$ is the toxicity rate constant; $k_{2}$ is the natural decay rate constant.

The rate of decay in the light produced by unchallenged bacteria can be described by eqn. (2):

$$
\frac{\mathrm{d} l_{\mathrm{U}, t}}{\mathrm{~d} t}=-k_{2} l_{\mathrm{U}, t}
$$

where $I_{\mathrm{U}, t}$ is the light intensity of the unchallenged bacteria at time $t$.

Integration and combination of eqn. (1) and (2) result in eqn. (3):

$$
\ln \left|\frac{l_{\mathrm{C}, t}}{l_{\mathrm{C}, 0}} \frac{l_{\mathrm{U}, 0}}{l_{\mathrm{U}, t}}\right|=-k_{1} t
$$

where $\left|\frac{l_{\mathrm{C}, t}}{l_{\mathrm{C}, 0}} \frac{l_{\mathrm{U}, 0}}{l_{\mathrm{U}, t}}\right|$ represents the corrected sample/blank ratio, or corrected light output (CLO).

By plotting the logarithm of the corrected light output (ln CLO) against time the toxic response rate constant, $k_{1}$, may be determined. Once $k_{1}$ has been determined it is possible to determine the time $\left(\mathrm{ET}_{50}\right)$ that is necessary for a particular sample to reduce the corrected light output by $50 \%$. ET 50 is effectively the half-life of the toxicity reaction.

When the light produced by challenged bacteria has been reduced by $50 \%$, due to the toxicity of the sample, $\ln \mid \frac{l_{\mathrm{C}, t} l_{\mathrm{U}, 0}}{l_{\mathrm{C}, 0}} \frac{l_{\mathrm{U}, t}}{\mid}=\ln 0.5$ and $t=\mathrm{ET}_{50}$, hence:

$$
\mathrm{ET}_{50}=\frac{\ln 0.5}{-k_{1}}
$$

The values of $k_{1}$ and $\mathrm{ET}_{50}$ are obviously concentration dependent. The greater the concentration of a toxin the greater the value of $k_{1}$, i.e. the steeper the slope of the ln CLO versus time plot, and the smaller the value of $\mathrm{ET}_{50}$. Determination of how $k_{1}$ and/or $\mathrm{ET}_{50}$ change with concentration allows the rate of change of toxicity with concentration to be established. In fact, the graph of $\mathrm{ET}_{50}$ versus concentration is a graph of $\mathrm{ET}_{50}$ versus $\mathrm{EC}_{50}$, where the $\mathrm{EC}_{50}$ is the effective concentration of the sample that reduces the light production of the bacteria by $50 \%$ in a test time of $\mathrm{ET}_{50}$. Such a graph can provide important information on how effective dilution of the sample would be in reducing toxicity. It is anticipated that this could form the basis of an additional and useful indicator in the assessment of chemical hazards. Furthermore, the values of $k_{1}$ and $\mathrm{ET}_{50}$ indicate the relative toxicity of the challenging substance when compared to the $k_{1}$ and $\mathrm{ET}_{50}$ values obtained with a wellcharacterised standard toxic compound.

Because the kinetic method relies on the rate of change in the light produced by the bacteria and not the absolute light intensities, interferences due to scatter and absorbance by the sample are not expected. As a consequence it is expected that coloured and turbid samples can be tested in exactly the same way as clear samples. Furthermore, as a great number of measurements is used for the determination of the toxicity rate constant and $\mathrm{ET}_{50}$ the method should be more robust than the commonly used approach and the Flash method, which was developed to defeat potential interference due to coloured and turbid test solutions. In this method, unlike the common approach, the bacteria are added to the sample dilutions and the percent inhibition is calculated using the maximum light intensity after the addition (instead of the intensity at time $0 \mathrm{~s}$ ) and the intensity measured after the incubation period. ${ }^{12,13}$ Another advantage of the kinetic method is the way the bacterial reagent is reconstituted, diluted and mixed with the test sample, which makes the reproducibility of the method much less dependent on the operator's ability, and suitable to be completely automated and used for remote screening.

\section{Materials and methods}

\section{Instrumentation}

The instrument used to apply the kinetic method was the inhouse built dual-channel prototype Toxos-3 described previously (Fielden et al., 1996). ${ }^{10}$ In this instrument the reagents are automatically mixed using miniature inert solenoid valves and gravity feed. The flow is then stopped and the light produced by challenged bacteria $\left(I_{\mathrm{C}, t}\right)$ and unchallenged bacteria $\left(I_{\mathrm{U}, t}\right)$ is measured simultaneously every $6 \mathrm{~s}$ (value averaged from 20 measurements taken at $100 \mathrm{~Hz}$ ) and plotted against time in real-time for $10 \mathrm{~min}$. $\ln$ CLO is also calculated 
Table 1 Table of reagents used

\begin{tabular}{|c|c|}
\hline Reagent & Supplier \\
\hline $\mathrm{NaCl}, 99+\%$ & Sigma-Aldrich Co. Ltd. \\
\hline $\mathrm{ZnCl}_{2}$, anhydrous powder, $99.999 \%$ & Sigma-Aldrich Co. Ltd. \\
\hline Microtox $^{\circledR}$ Acute Toxicity Testing Reagent (Vibrio fischeri strand NRRL B-11177) & AZUR Environmental Ltd. \\
\hline Reconstitution solution & AZUR Environmental Ltd. \\
\hline Polybead $^{\mathrm{B}} 6.0 \mu \mathrm{m}$ polystyrene microspheres dyed red, in water & Polysciences Inc. \\
\hline Polybead ${ }^{\circledR} \quad 6.0 \mu \mathrm{m}$ polystyrene microspheres dyed blue, in water & Polysciences Inc. \\
\hline Polybead $^{\circledR} 6.0 \mu \mathrm{m}$ polystyrene microspheres, in water & Polysciences Inc. \\
\hline Tropaeolin $\mathrm{O}$ & ACROS Organics \\
\hline
\end{tabular}

and plotted against time in real-time while $k_{1}$ is determined simultaneously using linear regression.

\section{Reagents}

Reagents used in these experiments are listed in Table 1. These were of the highest available purity.

\section{Procedure used for testing metal solutions}

Reconstitution and dilution of the bacteria was carried out immediately prior to use following the procedure described next. One Microtox ${ }^{\circledR}$ Acute Toxicity Testing Reagent vial was transferred from the freezer and was kept overnight at $2{ }^{\circ} \mathrm{C}$. All the materials (pipette tips, beakers, etc.) and solutions that were used to prepare the reagent were all pre-cooled to $2{ }^{\circ} \mathrm{C}$. The bacteria were then reconstituted with reconstitution solution as recommended by AZUR and diluted with $2 \% \mathrm{NaCl}$ to a concentration of 1 vial per $50 \mathrm{~mL}$.

Solutions osmotically adjusted to $2 \% \mathrm{NaCl}$, containing varying metal concentrations were tested at $15{ }^{\circ} \mathrm{C}$ in random order, to avoid trends due to reagent ageing. All the tests were carried out using a 1:1 sample:bacterial reagent ratio. The values of $k_{1}$ were determined using data obtained for $600 \mathrm{~s}$.

\section{Procedure for the assessment of the interference caused by turbidity and colour}

Reconstitution and dilution of the bacteria was carried out immediately prior to use. For the assessment of the effect of turbidity four Microtox ${ }^{\circledR}$ Acute Toxicity Testing Reagent vials were transferred from the freezer and were kept overnight at $2{ }^{\circ} \mathrm{C}$. All the materials (pipette tips, beakers, etc.) and solutions that were used to prepare the reagent were all pre-cooled to $2{ }^{\circ} \mathrm{C}$. The bacteria were then reconstituted with reconstitution solution as recommended by AZUR, mixed together and diluted with $2 \% \mathrm{NaCl}$ to a concentration of 1 vial per $30 \mathrm{~mL}$.

Solutions of $50 \mathrm{ppm}$ (parts per million) $\mathrm{Zn}^{2+}$ osmotically adjusted to $2 \% \mathrm{NaCl}$ containing microspheres from $0 \mathrm{mg} \mathrm{L}^{-1}$ to approximately $500 \mathrm{mg} \mathrm{L}^{-1}$ for each colour were then tested at $15{ }^{\circ} \mathrm{C}$ in random order, to avoid trends due to reagent ageing. All the tests were carried out using a 1:1 sample:bacterial reagent ratio. The values of $k_{1}$ were determined using data obtained for $350 \mathrm{~s}$, as after this time in some of the measurements the light level of the challenged bacteria was too low to be detected.

For the assessment of the effect of colour three vials of Microtox ${ }^{\circledR}$ Acute Toxicity Testing Reagent were reconstituted as described above and were diluted with $2 \% \mathrm{NaCl}$ to the same concentration of 1 vial per $30 \mathrm{~mL}$. Osmotically adjusted (to $2 \%$ $\mathrm{NaCl}$ ) solutions of $50 \mathrm{ppm} \mathrm{Zn}^{2+}$ and concentrations of tropaeolin $\mathrm{O}$ between 0 and $0.25 \mathrm{~g} \mathrm{~L}^{-1}$ were then tested at $15{ }^{\circ} \mathrm{C}$ in random order and in triplicate using a $1: 1$ sample:bacterial reagent ratio.

\section{Determination of scatter spectra of beads suspensions}

Scatter spectra of the suspensions containing the highest bead concentration of each colour were determined by measuring the intensity of the light scattered at $90^{\circ}$ by the suspensions in acrylic cuvettes $(10 \mathrm{~mm}$ in path length) using a Perkin Elmer Luminescence Spectrometer (LS 50 B).

\section{Results and discussion}

As had been reported by Fielden et al. ${ }^{10}$ when the reconstituted and diluted Vibrio fischeri were challenged with concentrations of metals that were toxic to them the light produced by the bacteria decreased exponentially with time following first order kinetics as shown by the linear relationship between ln CLO and time in, Fig. 1, for bacteria challenged with $50 \mathrm{ppm} \mathrm{Zn}^{2+}$ $\left(0.10 \mathrm{~g} \mathrm{~L}^{-1}\right.$ of $\left.\mathrm{ZnCl}_{2}\right)$ at $15{ }^{\circ} \mathrm{C}$.

This behaviour was not observed by Christofi and coworkers. ${ }^{14}$ The researchers challenged bacteria with concentrations of $\mathrm{ZnCl}_{2}$ between $5 \times 10^{-4}$ and $0.25 \mathrm{~g} \mathrm{~L}^{-1}$ and observed that the light produced by the bacteria increased during the first $15 \mathrm{~min}$. The light produced by the bacteria then decreased during the following $15 \mathrm{~min}$ and after that increased again. The researchers attributed the observed behaviour to hormesis response, an adaptive response, usually to subacute concentrations of a substance, which can either be directly induced by the substance or be the result of over-compensation to a disruption in homeostasis caused by the substance. ${ }^{15}$ However, the researchers observed this behaviour for concentrations well above that of the $\mathrm{IC}_{50}$ determined in their work $\left(0.408 \mathrm{mg} \mathrm{L}^{-1}\right)$.

One explanation for the difference in the observed behaviour could be the fact that Christofi's team used bacteria from shake cultures produced by them while the bacteria used for this work were the reconstituted and diluted (in $2 \% \mathrm{NaCl}$ ) freeze-dried bacteria obtained from AZUR Environmental Ltd. (now Strategic Diagnostics Inc.). Hence, the composition of the solution in which the bacteria were suspended would be expected to be quite different in both experiments. Also the age and the growth stage of the two sets of bacteria could be different. In general, the experimental conditions were quite different in the experiments carried out by the two groups and this could account for the difference in the observed behaviour of the bacteria.

In the present work it was also found that the light produced

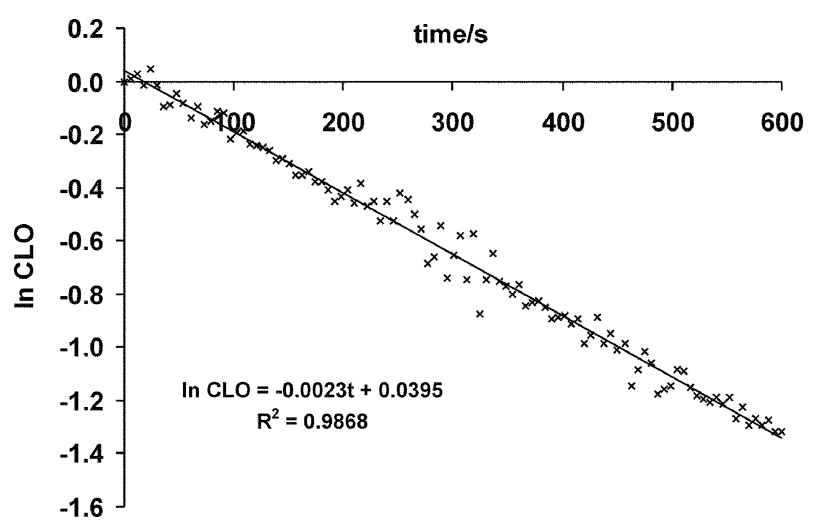

Fig. 1 Logarithm of the corrected light output (ln CLO) versus time

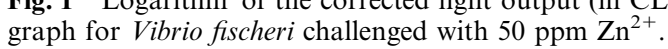




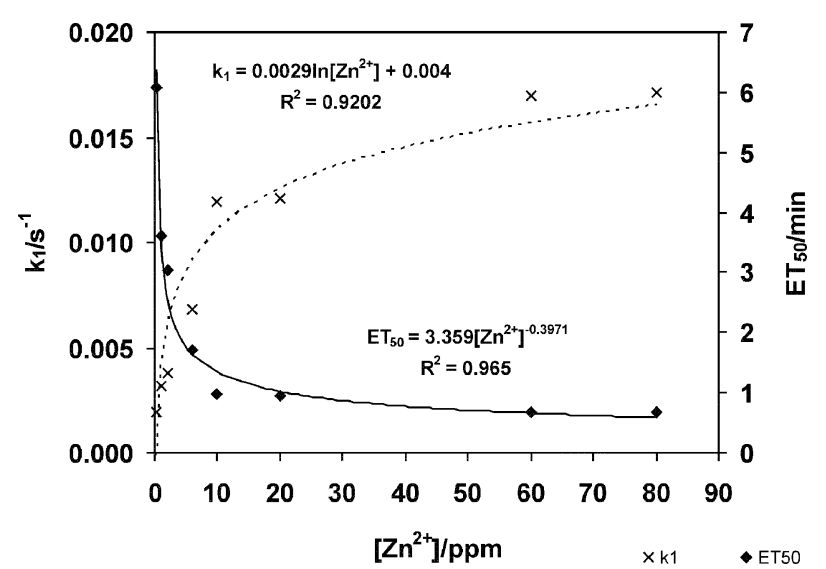

Fig. 2 Relationship between $k_{1}$ and $\mathrm{Zn}^{2+}$ concentration $\left(\left[\mathrm{Zn}^{2+}\right]\right)$, and the relationship between $\mathrm{ET}_{50}$ and $\mathrm{Zn}^{2+}$ concentration.

by unchallenged bacteria did not decrease following first order kinetics as previously assumed. ${ }^{10}$ Instead, the light produced remained constant for the first $c a .1000 \mathrm{~s}$. Only after this initial stationary period did the light produced by the bacteria decrease following first order kinetics. This was observed for different batches of bacteria and thus is not believed to be batch specific. This means that for the duration of the toxicity tests (usually $10 \mathrm{~min}$ or less) the light produced by the unchallenged bacteria remained virtually unchanged and thus the natural decay rate constant can be considered to be $k_{2} \approx 0 \mathrm{~s}^{-1}$. Consequently, blank correction is not necessary and eqn. (3) can be written as:

$$
\ln \frac{l_{\mathrm{C}, t}}{l_{\mathrm{C}, 0}}=-k_{1} t
$$

As no blank correction is necessary it is possible to use just one channel to determine toxicity. This would reduce the amount of bacterial reagent necessary per test and subsequently reduce the cost per test.

When several dilutions of a sample are tested, the relationship between $k_{1}$ and sample concentration, and the relationship between $\mathrm{ET}_{50}$ and sample concentration not only showed how toxicity changes with concentration but also provided useful information on the effect which dilution would have on the sample's toxicity. This might be a useful tool for the wastewater treatment industry to show what effect dilution would have on an inflowing stream that was too toxic to the activated sludge, and whether this would be a possible course of action. To illustrate this point, the relationship between $k_{1}$ and sample concentration, and the relationship between $\mathrm{ET}_{50}$ and sample concentration for $\mathrm{Zn}^{2+}, \mathrm{Pb}^{2+}$, and $\mathrm{Hg}^{2+}$ are shown in Fig. 2 to Fig. 4, respectively. Firstly, these figures show that as expected,

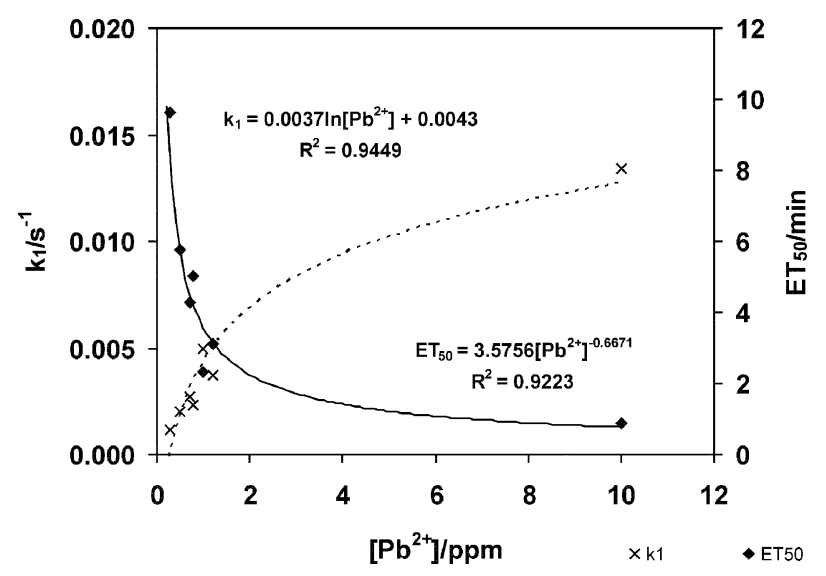

Fig. 3 Relationship between $k_{1}$ and $\mathrm{Pb}^{2+}$ concentration $\left(\left[\mathrm{Pb}^{2+}\right]\right){ }^{16}$ and the relationship between $\mathrm{ET}_{50}$ and $\mathrm{Pb}^{2+}$ concentration.

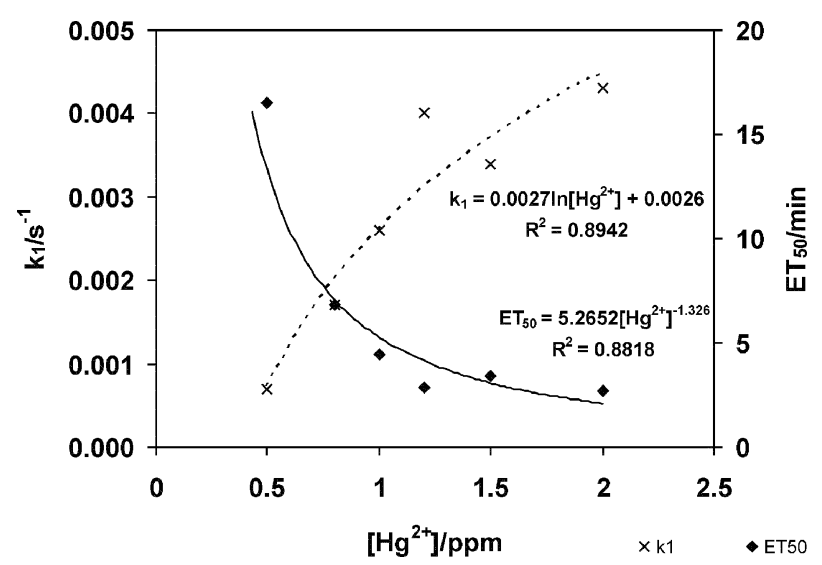

Fig. 4 Relationship between $k_{1}$ and $\mathrm{Hg}^{2+}$ concentration $\left(\left[\mathrm{Hg}^{2+}\right]\right),{ }^{16}$ and the relationship between $\mathrm{ET}_{50}$ and $\mathrm{Hg}^{2+}$ concentration.

the toxicity rate constant $k_{1}$ increases and that $\mathrm{ET}_{50}$ decreases with increased toxicity of the sample, i.e. metal concentration. In addition, if $\mathrm{Zn}^{2+}$ is taken as an example, Fig. 2 shows that if an $80 \mathrm{ppm}$ solution of $\mathrm{Zn}^{2+}$ were diluted to $40 \mathrm{ppm}, k_{1}$ and $\mathrm{ET}_{50}$ and consequently toxicity of the solution would hardly be affected. If, however, the solution were diluted to $2 \mathrm{ppm} k_{1}$ would be reduced from 0.0167 to 0.0034 and $\mathrm{ET}_{50}$ would increase from $41 \mathrm{~s}$ to $c a .3 \mathrm{~min}(182 \mathrm{~s})$.

The most important characteristic of the kinetic method which will be proven below, is however that unlike the commonly used Vibrio fischeri tests, the kinetic method is applicable to coloured and turbid samples and consequently could be used to test wastewaters.

\section{Effect of turbidity}

To investigate the hypothesis that turbidity caused by suspended solids did not interfere in the determination of toxicity when using the kinetic method, the toxicity of solutions of $50 \mathrm{ppm} \mathrm{Zn}^{2+}$ containing different concentrations of suspended particles was determined at $15{ }^{\circ} \mathrm{C}$. Turbidity was simulated using red, blue and white $6 \mu \mathrm{m}$ polystyrene beads. These beads were used because they are inert and therefore would not be toxic to the bacteria nor would they interfere in the toxicity of $\mathrm{Zn}^{2+}$.

The toxicity rate constants $\left(k_{1}\right)$ obtained for the solutions containing different concentrations of beads, shown in Fig.5, demonstrate that there was no obvious trend between $k_{1}$ and the concentration of beads ([Beads]). Furthermore, the coefficients of variation $(\mathrm{CV})$ of the values of $k_{1}$ for a $95 \%$ confidence interval for red, white and blue beads were $1.0 \%$ $\left(s=5.22 \times 10^{-5} \mathrm{~s}^{-1}, \bar{k}_{1}=4.98 \times 10^{-3} \mathrm{~s}^{-1}\right), 5.0 \%(s=$ 2.49 times; $\left.10^{-4} \mathrm{~s}^{-1}, \bar{k}_{1}=4.94 \times 10^{-3} \mathrm{~s}^{-1}\right)$, and $3.7 \%$

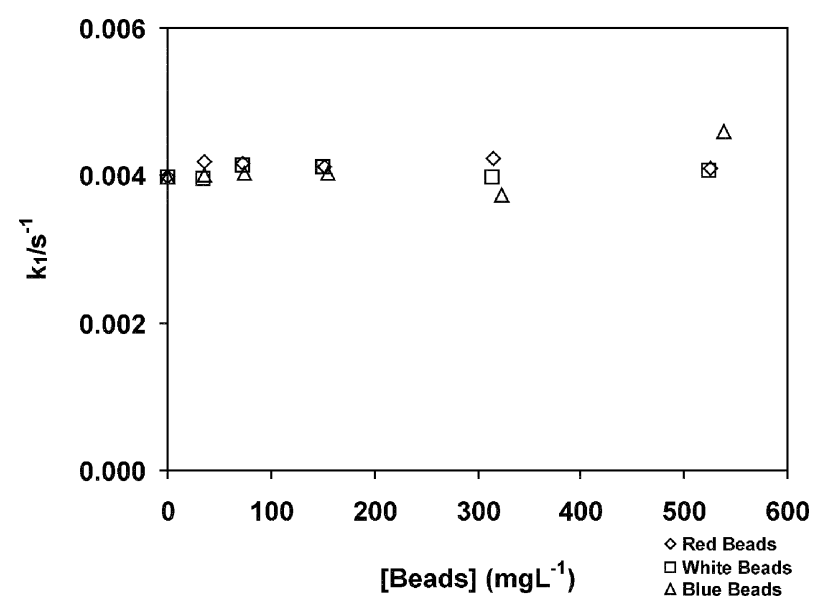

Fig. 5 Relationship between $k_{1}$ and bead concentration ([Beads]). 
$\left(s=1.94 \times 10^{-4} \mathrm{~s}^{-1}, \bar{k}_{1}=5.26 \times 10^{-3} \mathrm{~s}^{-1}\right)$, respectively. The coefficient of variation for a $95 \%$ confidence interval of all the values was $4.5 \%\left(s=2.29 \times 10^{-5} \mathrm{~s}^{-1}, \bar{k}_{1}=5.08 \times 10^{-3} \mathrm{~s}^{-1}\right)$. These results show that the presence of neither optically absorbing nor optically reflective suspended particles up to a concentration of $538 \mathrm{mg} \mathrm{L}^{-1}$ interfered in the determination of toxicity.

Thus it appeared that the kinetic measurement method was unaffected by suspended solids in the test solution. However, this conclusion could only be reached if the degree of scattering was known. To assess this, the ratio of light produced by the bacteria that is actually detected, i.e. the transmitted light $\left(T^{\prime}\right)$ was determined. This was done by determining the ratio $\left(Q_{x}\right)$ between the initial light output of the sample channel $\left(I_{\mathrm{C}, 0, x}\right)$ (i.e. the light detected) and the initial light output of the blank channel $\left(I_{\mathrm{U}, 0, x}\right)$ (i.e. the light produced by the bacteria). To obtain $T^{\prime}, Q_{x}$ was then corrected for bacterial and channel response variations as shown in eqn (7) by dividing $Q_{x}$ by the same ratio obtained for the clear solution $\left(Q_{0}\right)$.

$$
Q_{x}=\frac{l_{\mathrm{C}, 0, x}}{l_{\mathrm{U}, 0, x}}
$$

Where: $I_{\mathrm{C}, 0, x}$ is the initial light output of the sample channel when testing a suspension containing $\mathrm{x} \mathrm{mgL}^{-1}$ of beads. $I_{\mathrm{U}, 0, x}$ is the initial light output of the blank channel when testing the same suspension.

$$
T^{\prime}=\frac{Q_{x}}{Q_{0}}
$$

The results of the experiment for solutions of different bead concentrations are shown in Fig. 6. These results show that there was no significant difference between the light transmitted $\left(T^{\prime}\right)$ by the suspensions of different bead concentrations. This suggests that either bead suspensions in the given concentration range did not scatter the light produced by the bacteria significantly or the Toxos-3 instrument is not susceptible to scattering interferences. To elucidate this further the scattering properties of the bead suspensions was evaluated by determining the scatter spectra of the suspensions containing the highest concentration of beads of each colour. The scatter spectra of the bead-containing solutions and the Vibrio fischeri emission spectrum shown in Fig. 7 confirm that the beads do scatter light in the wavelength band emitted by the bacteria. The strongest scatter is observed for white and blue beads, yet even these do not appear to have any influence on the measured rate constant.

The reason for not observing interferences due to scatter is thought to be the design of the flow cell chamber whose walls are covered with aluminium foil and thus reflect the scattered light back into the photomultiplier as is schematically shown in Fig. 8. Although from this experiment it could not be said that

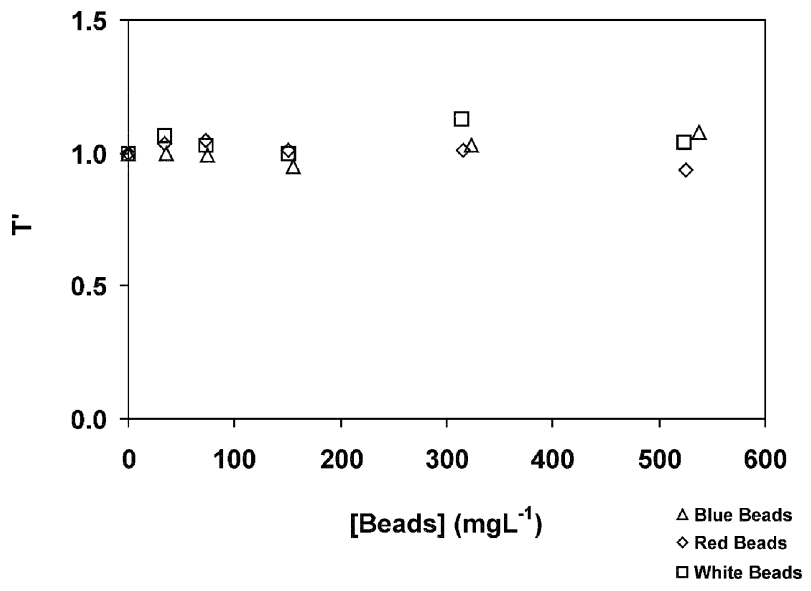

Fig. 6 Light transmitted $\left(T^{\prime}\right)$ by suspensions containing beads.

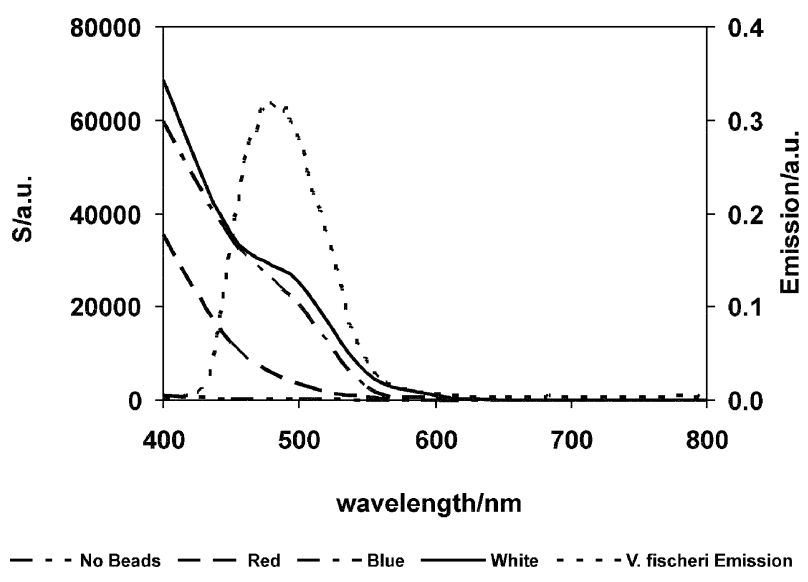

Fig. 7 Scatter $(S)$ spectra of the suspensions containing greatest concentration of beads of each colour, and Vibrio fischeri emission spectrum.

turbidity does not interfere in the determination of toxicity when using the kinetic method, it can be said that the test using this instrumental design does not suffer interferences due to turbidity. Consequently, turbid samples can be tested in the same way as clear samples without the need for pre-treatments like filtration (which are laborious and affect the representativity of the sample) or complicated correction procedures. Furthermore, the efficient light collection of the instrument widens the range of samples that can be tested as the amount of light that is detected is a limiting factor for the suitability of a sample to be tested using Vibrio fischeri.

The hypothesis that kinetic methods do not suffer from interference effects such as those from scattering or absorption in coloured solutions is easier to demonstrate in the latter case as the light collection optical system shown in Fig. 8 would not alleviate absorbance effects as it does for scattering effects.

\section{Effect of colour}

To investigate the hypothesis that colour does not interfere in the determination of toxicity when using the kinetic method solutions of $50 \mathrm{ppm} \mathrm{Zn}^{2+}$ containing concentrations of the food-dye tropaeolin $\mathrm{O}$ between 0 and $0.25 \mathrm{~g} \mathrm{~L}^{-1}$ were analysed. Tropaeolin $\mathrm{O}$ absorbs some of the wavelengths emitted by Vibrio fischeri as is shown by the overlapping of the Vibrio fischeri emission spectrum and the tropaeolin $\mathrm{O}$ absorbance spectrum as shown in Fig. 9.

The results of the analysis are shown in Fig. 10 as a function of $T^{\prime}$ which was calculated using eqn (7). There is no trend between the toxicity rate constant and $T^{\prime}$ even when $52 \%$ of the light produced by the bacteria is absorbed by the sample $\left(T^{\prime}=\right.$ 0.48 ). Furthermore, the coefficient of variation of the $k_{1}$ values

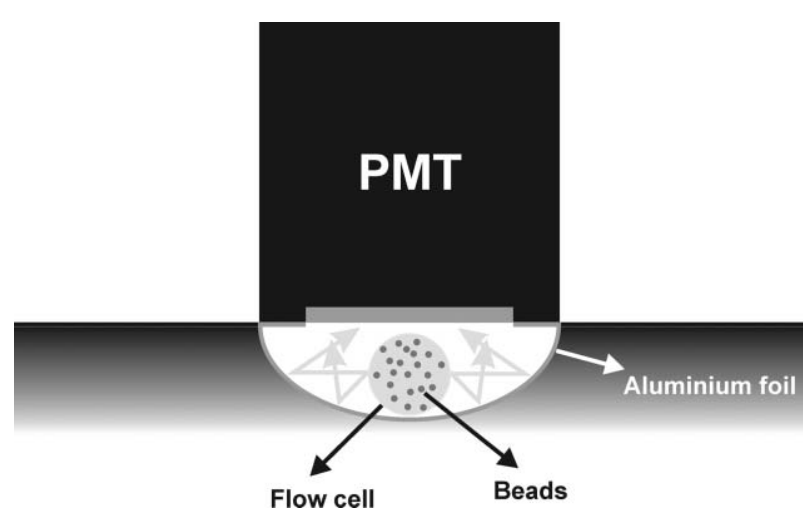

Fig. 8 Schematic representation of the flow cell chamber, and scattered light being reflected back to the photomultiplier by the flow chamber wall. 


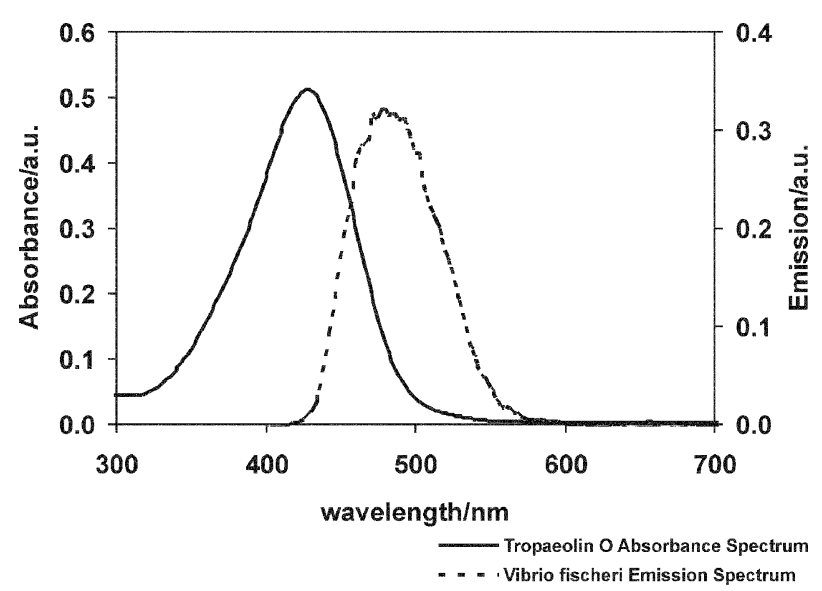

Fig. 9 Vibrio fischeri emission spectrum and tropaeolin $\mathrm{O}$ absorbance spectrum

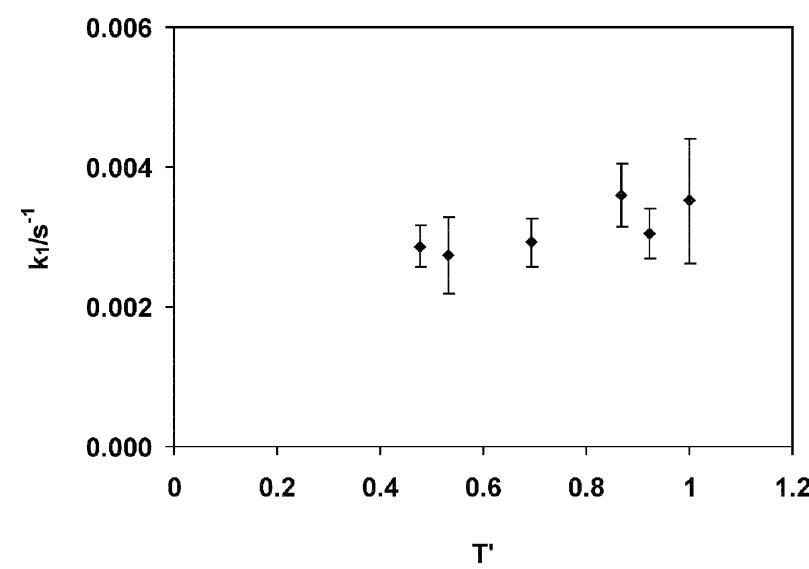

Fig. 10 Variation of toxicity rate constant $\left(k_{1}\right)$ with transmitted light $\left(T^{\prime}\right)$ for solutions of $50 \mathrm{ppm} \mathrm{Zn}^{2+}$ and varying concentrations of tropaeolin $\mathrm{O}$.

obtained for the different dye concentrations was 12\% $(s=$ $3.72 \times 10^{-4} \mathrm{~s}^{-1}, \bar{k}_{1}=3.15 \times 10^{-3} \mathrm{~s}^{-1}$ ). This leads us to conclude that the presence of colour does not interfere in the determination of the rate constant and hence toxicity when using the kinetic method. Consequently, coloured samples can be tested like clear samples without the need for laborious correction procedures. This obviously assumes there is still sufficient light remaining to allow the measurement to be carried out.

Besides showing that the colour does not interfere in the determination of toxicity when the kinetic method is used, these results also provide support to the hypothesis that the kinetic method is free from interference due to turbidity of the sample. There are however conditions where this independence from colour interferences would be expected to fail for example if the absorbance at the interfering wavelength were to change significantly during the measurement time thus apparently altering the rate of decay of the light output from the measurement cell during the determination. Although changes in absorbance are unlikely with the currently used instrument and analysis times, the same cannot be said for turbidity changes where suspended solids could settle during the measurement cycle. In respect of the latter, the independence of the measured intensity from scatter as a consequence of careful instrument design as demonstrated above is important.

\section{Conclusions}

In this paper we have shown that the kinetic method of toxicity determination using the Toxos-3 instrument and the widely used marine luminescent bacterium Vibrio fischeri as test organism, which is easy to use and provides an inexpensive rapid response, does not suffer from interferences due to colour or turbidity of the sample. This implies that turbid and coloured samples, like wastewaters, can be tested like clear samples. It was also shown that the design of the instrument allows efficient collection of the light scattered by particles present in the sample so that the detected light was not reduced due to scatter. This is of great importance as bioluminescence tests are limited by the need for sufficient light to be detected for the test to be carried out.

These characteristics make the kinetic method potentially useful for remote toxicity screening of wastewaters without the need to determine whether a sample is coloured and/or turbid or the need for correction procedures. Furthermore, because the sample dilutions and the mixing of reagent are all automated the precision of the test does not depend on operator ability.

Also, it was shown that for the duration of the tests the natural light decay is negligible and consequently that a blank channel would not be necessary, thus halving the amount of bacterial reagent used and reducing the cost of the tests.

Furthermore, the independence of the rate constant on the initial measured light output combined with independence from scatter and colour interferences also opens up the possibility of development of a miniaturised toxicity test using simple dip-stick technology. There would however be one remaining potential drawback for such an application of the kinetic method and that would be the need for precise temperature control as is common with all chemical kinetic measurements.

\section{Acknowledgements}

The authors would like to thank UMIST Graduate School and the Fundação para a Ciência e Tecnologia (PRAXIS XXI) for funding this work.

\section{References}

1 C. Genner and R. Kruger, North West. Branch Pap.-Inst. Chem. Eng., 1995, 2, 1.

2 J. E. Burgess, P. J. Longhurst, J. Quarmby and T. Stephenson, Technovation, 2000, 20, 37

3 On World Wide Web: http://cfpub1.epa.gov/npdes/wqbasedpermitting/wet.cfm, Environmental Protection Agency, Ariel Rios Building, 1200 Pennsylvania Avenue, N.W., Washington, DC 20460, USA.

4 J. E. Burgess, P. Longhurst, J. Quarmby and T. Stephenson, Technovation, 2000, 20, 313 .

5 M. Castillo, M. C. Alonso, J. Riu, M. Reinke, G. Klöter, H. Dizer, B. Fischer, B. Hansen and D. Barceló, Anal. Chim. Acta, 2001, 426, 265.

6 D. J. B. Dalzell, S. Alte, E. Aspichueta, A. de la Sota, J. Etxebarria, M. Gutierrez, C. C. Hoffmann, D. Sales, U. Obst and N. Christofi, Chemosphere, 2002, 47, 535.

7 M. Farré, G. Klöter, M. Petrovic, M. C. Alonso, M. J. L. de Alda and D. Barceló, Anal. Chim. Acta, 2002, 456, 19.

8 V. L. K. Jennings, M. H. Rayner-Brandes and D. J. Bird, Water Res., 2001, 35, 3448

9 Beckman Microtox ${ }^{(i)}$ System Operating Manual, Beckman Instruments Inc., Microbics Operations, Carlsbad, CA.

10 P. R. Fielden, T. McCreedy, R. D. Snook and B. J. Treves Brown, Anal. Commun., 1996, 33, 335 .

11 F. H. Johnson, H. Eyring and B. J. Storer, The Theory of Rate in Processes in Biology and Medicine, Wiley, New York, 1974, pp. $461-471$.

12 J. Lappalainen, R. Juvonen, J. Nurmi and M. Karp, Chemosphere, 2001, 45, 635.

13 J. Lappalainen, R. Juvonen, K. Vaajasaari and M. Karp, Chemosphere, 1999, 38, 1069.

14 N. Christofi, C. Hoffmann and L. Tosh, Ecotoxicol. Environ. Saf., 2002, 52, 227.

15 E. J. Calabrese and L. A. Baldwin, Hum. Exp. Toxicol., 2002, 21, 91.

16 M. Ryan, MSc Dissertation, UMIST, UK, 1997. 